Open Access to Pharmaceutical and Medical Research
uDDT* s\%

Open 1 Access

Review Article

\title{
SGLT2 Inhibitors: A Noval Therapuetic Agent in the Treatment of Diabetic Kidney Disease
}

\author{
K.V. Musaina Thasneem*, K. Sreejith, B. Athulnadh, Namitha Maniyan, Cherakkulath C Neena, P.P \\ Muhamed Faris \\ Department of Pharmacy Practice, College of Pharmaceutical Sciences, Govt. Medical College, Kozhikode, Kerala, 673008, India
}

\begin{abstract}
Sodium Glucose Co-transporter2 inhibitors are one of the latest anti diabetic drugs that are approved by USFDA. It include Dapagliflozin, Canagliflozin , Ipragliflozin,Empagliflozin, Tofogliflozin,and Luscogliflozin. They act by inhibiting tubular reabsorption of glucose in kidney and increasing urinary excretion of glucose. SGLT2 inhibitors reduce the workload of the proximal tubules and improve tubulointerstitial hypoxia, and allow fibroblasts to start normal erythropoietin production, and thereby exhibit renoprotection.These drugs have beneficial role in the reduction of HbA1c, cardiovascular risk factors and proteinuria. Use of SGLT2 inhibitor is contraindicated in patients with e stimated GFR less than $30 \mathrm{~mL} / \mathrm{min}$ or End stage renal failure Genitourinary infections are most common adverse effects associated with these drugs, predominantly in female.
\end{abstract}

Keywords: Diabetes Mellitus, Diabetic Nephropathy, Hyperfiltration, Natriuresis, Macroalbuminuria, Endothelial dysfunction, Intraglomerular filtration, ketoacidosis, amputations, apoptosis

Article Info: Received 06 Sep 2020; Review Completed 14 Oct 2020; Accepted 22 Oct 2020; Available online 15 Nov 2020

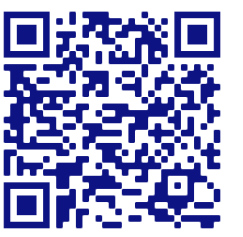

Cite this article as:

Thasneem KVM, Sreejith K, Athulnadh B, Maniyan N, Neena CN, Faris PPM, SGLT2 Inhibitors: A Noval Therapuetic Agent in the Treatment of Diabetic Kidney Disease, Journal of Drug Delivery and Therapeutics. 2020; 10(6):203-206 http://dx.doi.org/10.22270/jddt.v10i6.4532

K.V. Musaina Thasneem, Department of Pharmacy Practice, College of Pharmaceutical Sciences, Govt. Medical College, Kozhikode, Kerala, 673008, India

\section{INTRODUCTION}

Diabetes Mellitus is a complex, chronic disease that requires multiple drugs as the disease progresses. As per the current guidelines, Metformin is the first line pharmacological agent for treatment of non-insulin dependent Diabetes mellitus. Multiple second line therapy is available for those patients who do not respond to monotherapy. Selection of multiple therapies should be based on patient specific and drug specific factors. . In 2030, Diabetes Mellitus will be the seventh leading cause of death. Non-insulin dependent diabetes is characterized by $\beta$ cell destruction and insulin resistance which occur in liver and muscles. Numerous changes takes place in organs like fat cells, $\alpha$ cell, kidney, gastrointestinal tract and brain 1

Diabetic nephropathy is present in $40 \%$ of non-insulin dependent diabetes patients. Risk factors for Diabetic nephropathy include albuminuria, anemia, dyslipidemia, hyperglycemia, hypertension and lifestyle factors like obesity, smoking. Early identification and treatment of these factors can slow the progression or prevent Diabetic kidney disease. In Western world, Diabetes is the most common cause of End Stage Renal failure. Diabetic kidney disease is defined as the presence of macro albuminuria (albumin $\geq$ $300 \mathrm{mg} / \mathrm{dl}$ ) and decreased Glomerular Filtration Rate. Diabetic Nephropathy is characterized by endothelial dysfunction, coagulation abnormalities, vascular inflammation and high decrease in nocturnal blood pressure and increased risk for cardiovascular disease. Diabetic Nephropathy can be categorized in to 2 stages based on Urinary Albumin Excretion [UAE] values. If the UAE value is $30-299 \mathrm{mg} / 24 \mathrm{~h}$, then it is micro albuminuria and if the same is $\geq 300 \mathrm{mg} / 24 \mathrm{~h}$,then it is macro albuminuria. Diabetes causes alterations in kidney structure. Glomerulosclerosis is associated with increase in width of glomerular basement membrane, diffuse masangial sclerosis, micro aneurysm along with tubular and interstitial changes ${ }^{2}$ 


\section{SGLT2 INHIBITORS}

Sodium Glucose Co-transporter2 inhibitors are one of the latest anti diabetic drugs that are approved by USFDA. SGLT2 inhibitor is expressed predominantly in S1 segment of proximal convoluted tubule of kidney. It is the major transporter that mediates renal glucose reabsorption. The first SGLT2 inhibitor was Phlorizin. It was a naturally occurring compound that causes gastrointestinal symptoms due to its non-selective nature. So drugs that specifically inhibit SGLT2 have been developed. It include Dapagliflozin, Canagliflozin , Ipragliflozin,Empagliflozin, Tofogliflozin,and Luscogliflozin. Among SGLT2 inhibitors, Canagliflozin, Dapagliflozin, Empagliflozin are currently available in United States. In people with Diabetes, reabsorption of glucose is less when compared to those without Diabetes. SGLT2 inhibitors act by inhibiting tubular reabsorption of glucose in kidney and increasing urinary excretion of glucose, thereby promote glycemic control and weight loss. These drugs are contraindicated in patients with $\mathrm{GFR}<45 \mathrm{ml} / \mathrm{min}^{3}$

\section{- Dapagliflozin}

Dapagliflozin is rapidly absorbed and reach peak plasma concentration within 2 hours after oral administration. It exhibit $78 \%$ oral bioavailability. Uridine diphosphate glucuronosyltransferase (UGT)1A9 in both liver and kidney metabolise Dapagliflozin. It is proven that Dapagliflozin is efficacious in enhancing glycemic control both as a single agent and in combination with Canagliflozin.4 year administration of Dapagliflozin and Metformin combination produced sustained decrease in blood glucose level with least adverse effects when compared to combination of Glimepiride and Metformin. Dapagliflozin increased insulin mediated tissue glucose destruction $18 \%$ in 2 week treatment and resulted in increased endogenous glucose production. Dapagliflozin dose range is $5-10 \mathrm{mg}$. 5-mg dose is recommended for patients with impaired hepatic function. Dose adjustment is not required in mild to moderate hepatic dysfunction. 5mg dose is recommended in acute hepatic disease. 4

\section{- Canagliflozin}

Canagliflozin reach peak plasma levels within 1 to 2 hour after oral administration and reach steady state after 4-5 days. It exhibit $65 \%$ oral bioavailability. UGT1A9 and UGT2B4 metabolise Canagliflozin. It is proven that Canagliflozin is efficacious in enhancing glycemic control both as a single agent and in combination with Dapagliflozin. Dose range is $100-300 \mathrm{mg}$ once daily before the first meal of the day. The dose can be increased to $300 \mathrm{mg}$ in those who do not respond adequately, provided that the renal function is normal. It is not recommended in patients with eGFR less than $45 \mathrm{ml} / \mathrm{min}^{5}$

\section{- Empagliflozin}

Empagliflozin reach peak plasma levels within 1-3 hour after oral administration. Dose range is $10-25 \mathrm{mg}$ once daily. Dose adjustment is required in patients with creatinine clearance less than $60 \mathrm{ml} / \mathrm{min}$ and it is contraindicated in patients with creatinine clearance less than $45 \mathrm{ml} / \mathrm{min}$. Dose adjustment is not required in patients with hepatic insufficiency 6

\section{- Ipragliflozin}

Ipragliflozin is approved in Japan for the treatment of noninsulin dependent Diabetes, either alone or in combination with Pioglitazone, Metformin, Sulfonylureas, Dipeptidyl peptidase 4 inhibitor, or with Nateglinide. Dose range is 100 $300 \mathrm{mg}$ once daily. It exhibit $65 \%$ oral bioavailability.It is not recommended in patients with estimated GFR less than 45 $\mathrm{ml} / \mathrm{min}^{7}$

\section{- Tofogliflozin}

Tofogliflozin is approved in Japan in 2014 for treatment of non-insulin dependent Diabetes, either alone or in combination with other oral antidiabetic drugs. Combination therapy of Insulin and Tofogliflozin reduced HbA1c and post prandial blood glucose in 16 week. It also caused slight reduction in $\mathrm{BP}$ and body weight ${ }^{8}$

\section{BENEFITS OF SGLT2 INHIBITORS}

\section{- HbA1C}

HbA1C levels are reduced by Canagliflozin, Dapagliflozin and Empagliflozin ${ }^{9}$

\section{- $\quad$ Lipid Profile}

SGLT2 inhibitors produce cardiovascular benefit by reducing cardiovascular risk factors. Dyslipidemia is a major comorbidity of non-insulin dependent diabetes mellitus which cause cardiovascular mortality and morbidity. Studies found that Dapagliflozin reduce LDL cholesterol and increase HDL Cholesterol. Empagliflozin produce small raise in HDL and LDL cholesterol. Thus it facilitates fecal excretion of LDL cholesterol and macrophage derived cholesterol ${ }^{10}$

\section{- Body Weight}

Studies found that Dapagliflozin reduce body weight from 1$3 \mathrm{~kg}$ compared to placebo. Canagliflozin 100 or $300 \mathrm{mg}$ can cause dose dependent weight loss. Empagliflozin cause less adiposity.10

\section{- $\quad$ Blood Pressure}

Reduction of arterial blood pressure causes reduction of cardiovascular mortality and morbidity in diabetic patients. In non-insulin dependent Diabetic patients and hypertensive patients, Dapagliflozin reduce mean systolic blood pressure. Empagliflozin reduce both systolic and diastolic blood pressure without increase in heart rate ${ }^{10}$

\section{- Albuminuria}

SGLT2 Inhibitors Produce Renoprotective Effects In NonInsulin Dependent Diabetic Patients. In Patients With NonInsulin Dependent Diabetes Mellitus And Hypertension Receiving Renin Angiotensin Blockers, Dapagliflozin And Canagliflozin Reduce Albuminuria. In Patients with Micro or Macroalbuminuria, Empagliflozin Reduce Urine Albumin Creatinine Ratio, Thereby Produce Renal Protection.10

\section{- Cardiovascular Effect}

In Diabetic patients,cardiac inflammation leads to cardiomyopathy. SGLT2 inhibitors directly reduce cardiac inflammation. SGLT2 inhibitors cause natriuresis which cause decrease in plasma volume and blood pressure. Thereby they cause reduction in cardiac preload and afterload. This occur without any change in heart rate.Dapagliflozin can stimulate pancreatic alpha cells and cause secretion of glucagon which increase ketogenesis and thus improve cardiac contractility. Ketone bodies increase mitochondrial biogenesis and are associated with antiarrythmia.SGLT2 inhibitors act as antioxidant and reduce cardiac oxidative stress 11 Empagliflozin attenuate cardiomyocyte apoptosis in dose dependent manner. SGLT2 inhibitors may inhibit $\mathrm{Na} / \mathrm{k}$ exchanger that is upregulated in heart failure patients ${ }^{12}$. A study with Empagliflozin shows decrease in calcium and sodium levels of myocardial cytoplasm and raise in mitochondrial calcium levels. In rat models,administration dapagliflozin produced cardiac 
antifibrotic effect by reducing collagen synthesis and inhibiting myofibroblast differentiation. Both metabolic and hemodynamic actions of SGLT2 inhibitors improve diabetic cardiomyopathy, this results in cardioprotection 13 .

\section{- Renoprotection}

The entry of glucose in to kidney tubular cells is the basis for the development of diabetic nephropathy.It cause a histological change which is called as tubulointerstitial fibrosis. It involves both proximal tubular cell (PTCs) basement membrane alterations and interstitial fibrosis. The profibrotic and inflammatory effects in PCTs are due to elevated glucose level and profibrotic cytokine transforming growth factor $\beta$ (TGF $\beta$ ). The tubulointerstitial inflammation caused by elevated glucose level can be reversed by SGLT2 inhibition. ${ }^{14}$

SGLT2 inhibitors exhibit Reno protection indirectly by reducing reabsorption of glucose in kidney, thereby reduce blood glucose level and body weight.SGLT2 inhibitors specifically cause alterations in renal hemodynamics and reduction in intra glomerular pressure and reduce diabetesassociated hyperfiltration, tubular hypertrophy, and tubular toxicity of glucose to directly protect kidney. SGLT2 inhibitors reduce albuminuria, serum uric acid, systolic blood pressure (SBP) by mild natriuresis, osmotic dieresis, afferent arteriole constriction, and weight loss . Hematocrit and erythropoietin is increased due to diuresis. SGLT2 inhibitors reduce the workload of the proximal tubules and improve tubulointerstitial hypoxia, and allow fibroblasts to start normal erythropoietin production, and thereby exhibit renoprotection 15 .

Use of SGLT2 inhibitor is contraindicated in patients with estimated GFR less than $30 \mathrm{~mL} / \mathrm{min}$ or End stage renal failure. When Empagliflozin 10 or $25 \mathrm{mg}$ is given for noninsulin dependent diabetic patients with mild to moderate renal impairment, a small decrease in eGFR and albuminuria has occurred. There was a change in albumin to creatinine ratio and blood urea nitrogen in stage 3 CKD patients treated with SGLT2 inhibitors.SGLT2 inhibitor treatment for 26 weeks cause reduction in above parameters along with increase in serum potassium and magnesium in those patients. ${ }^{16}$

The main route of elimination of Canagliflozin is in faeces (50\%), whereas Dapagliflozin is in urine (75\%). Approximately 20\% of Empagliflozin is excreted unchanged in urine. The glucose-lowering efficacy of SGLT2 inhibitors depend on renal function.17

\section{RISKS OF SGLT2 INHIBITORS}

Nausea, fatigue, polyuria, polydypsia are common adverse effects. Serious adverse effects are as follows:

\section{- Increased Risk for Amputations}

Studies shown that Canagliflozin doubled the risk for leg and foot amputations in participants when compared to placebo. So Canagliflozin should be given with caution to the patient with past history of neuropathy, foot ulceration or vacular diseases ${ }^{9}$

\section{- Genito-Urinary Infections}

SGLT2 inhibitors produce urinary tract infections and vulvovaginitis due to glycosuria. Infections are most common in females.9,10

\section{- $\quad$ Acute Renal Injury}

The mechanism of renal injury is unknown. Studies shown that some patients experience renal failure within one month of starting medication. Symptoms improved when the drug was discontinued. 9

\section{- Hypotension}

Since SGLT2 inhibitors cause reduction in systolic and diastolic blood pressure, they cause postural hypotension and dizziness especially in elder patients and those who are taking loop diuretics. So these drugs should be prescribed with caution in elderly patients.9,10

\section{- Bone Fracture}

Studies show that long term Canagliflozin use cause increased risk for bone fracture due to decreased bone mineral density.SGLT2 inhibitors cause raise in serum phosphate levels, thus cause increase in Parathyroid hormone and fibroblast growth factor which promote phosphatouria that have opposing action on vitamin D metabolism.9,10

\section{- Diabetic Ketoacidosis}

In 2015 May, FDA issued a warning that SGLT2 inhibitor use may increase the risk for ketoacidosis in both juvenile and non-insulin dependent Diabetes mellitus patients. In a study of type 1 Diabetes mellitus patients treated with Empagliflozin for a period of 8 weeks were withdrawn from study because of Diabetic ketoacidosis. Two patients receiving canagliflozin for Type 1 Diabetes mellitus described multiple episodes of ketoacidosis even though they had never hospitalized for diabetic ketoacidosis before starting SGLT2 inhibitor therapy. Approximately 20 SGLT2 inhibitor treated patients were reported to have ketoacidosis and reported in FDA adverse event reporting system. At least one SGLT2 inhibitor was reported to cause dose dependent raise in acetoacetate and $\beta$-hydroxy butyrate levels. Further investigations were done to find out pathogenesis of ketoacidosis. ${ }^{18}$ Investigations found that the dose of insulin administered to Diabetic patients were decreased due to glucose lowering property of SGLT2 inhibitor to reduce the risk of hypoglycemia. This reduction in insulin level may increase the rate of lipolysis in adipose tissue and ketogenesis in the liver which increase the level of circulating ketone bodies ${ }^{19}$. Another suggested mechanism is that SGLT2 inhibitor may cause reduced excretion of ketone bodies synthesized in the body. 20

\section{EFFICACY OF SGLT2 INHIBITORS}

The action of SGLT2 inhibitors is independent on insulin ,so they can be used at all stages of type 2 Diabetes mellitus because of lower risk for hypoglycemia. Because of diuretic effect they also reduce blood pressure. Weight reduction of around $2 \mathrm{~kg}$ is observed due to glycosuric effect. 21

\section{DRUG INTERACTIONS}

- Concomitant administration of SGLT2 inhibitors with loop diuretics cause volume depletion.

- UDP glucuronyltransferase inducers(eg:Rifampicin, phenytoin, phenobarbitone, ritonavir) increase the metabolism of Canagliflozin ,so dose should be increased from 100-300 mg. ${ }^{22}$

\section{REFERENCES}

1) Chao EC, Henry RR. SGLT2 inhibition-a novel strategy for diabetes treatment.Nature Reviews Drug Discovery. 2010 Jul; 9(7):551

2) Cooper ME. Pathogenesis, prevention, and treatment of diabetic nephropathy. The Lancet. 1998 Jul 18; 352(9123):213-9

3) Rieg T, Masuda T, Gerasimova M, Mayoux E, Platt K, Powell DR, Thomson SC, Koepsell H, Vallon V. Increase in SGLT1-mediated 
transport explains renal glucose reabsorption during genetic and pharmacological SGLT2 inhibition in euglycemia. American Journal of Physiology-Renal Physiology. 2013 Nov 13; 306(2):F188-93.

4) Kasichayanula S, Liu X, LaCreta F, Griffen SC, Boulton DW. Clinical pharmacokinetics and pharmacodynamics of dapagliflozin, a selective inhibitor of sodium-glucose co-transporter type 2 . Clinical pharmacokinetics. 2014 Jan 1; 53(1):17-27.

5) Devineni D, Curtin CR, Polidori D, Gutierrez MJ, Murphy J, Rusch S, Rothenberg PL. Pharmacokinetics and pharmacodynamics of canagliflozin, a sodium glucose co-transporter 2 inhibitor, in subjects with type 2 diabetes mellitus. The Journal of Clinical Pharmacology. 2013 Jun; 53(6):601-10

6) Macha S, Mattheus M, Halabi A, Pinnetti S, Woerle HJ, Broedl UC. Pharmacokinetics, pharmacodynamics and safety of empagliflozin, a sodium glucose cotransporter2 (SGLT2) inhibitor, in subjects with renal impairment. Diabetes, Obesity and Metabolism. 2014 Mar; 16(3):215-22

7) Zhang W, Krauwinkel WJ, Keirns J, Townsend RW, Lasseter KC, Plumb L, Kadokura T, Ushigome F, Smulders R. The effect of moderate hepatic impairment on the pharmacokinetics of ipragliflozin, a novel sodium glucose co-transporter 2 (SGLT2) inhibitor. Clinical drug investigation. 2013 Jul 1; 33(7):489-96.

8) Schwab D, Portron A, Backholer Z, Lausecker B, Kawashima K. A novel double-tracer technique to characterize absorption, distribution, metabolism and excretion (ADME) of $\left[\begin{array}{ll}14 & \mathrm{C}\end{array}\right]$ tofogliflozin after oral administration and concomitant intravenous microdose administration of [13 C] tofogliflozin in humans.Clinical pharmacokinetics. 2013 Jun 1; 52(6):463-73

9) Scheen AJ. SGLT2 inhibitors: benefit/risk balance. Current diabetes reports. 2016 Oct $1 ; 16(10): 92$.

10) Lupsa BC, Inzucchi SE. Use of SGLT2 inhibitors in type 2 diabetes: weighing the risks and benefits. Diabetologia. 2018 Oct 1; 61(10):2118-25.

11) Verma S, McMurray JJ. SGLT2 inhibitors and mechanisms of cardiovascular benefit: a state-of-the-art review. Diabetologia. 2018 Oct $1 ; 61(10): 2108-17$.

12) Foote C, Perkovic V, Neal B. Effects of SGLT2 inhibitors on cardiovascular outcomes. Diabetes and Vascular Disease Research. 2012 Apr; 9(2):117-23.
13) Ryan PB, Buse JB, Schuemie MJ, DeFalco F, Yuan Z, Stang PE, Berlin JA, Rosenthal N. Comparative effectiveness of canagliflozin, SGLT2 inhibitors and non-SGLT2 inhibitors on the risk of hospitalization for heart failure and amputation in patients with type 2 diabetes mellitus: a real-world metaanalysis of 4 observational databases (OBSERVE-4D). Diabetes, Obesity and Metabolism. 2018 Nov; 20(11):2585-97..

14) Vallon $V$, Thomson SC. Targeting renal glucose reabsorption to treat hyperglycaemia: the pleiotropic effects of SGLT2 inhibition. Diabetologia. 2017 Feb 1; 60(2):215-25.

15) Chino Y, Samukawa Y, Sakai S, Nakai Y, Yamaguchi JI, Nakanishi T, Tamai I. SGLT2 inhibitor lowers serum uric acid through alteration of uric acid transport activity in renal tubule by increased glycosuria. Biopharmaceutics\& drug disposition. 2014 Oct; 35(7):391-404.

16) Schernthaner G, Mogensen CE, Schernthaner GH. The effects of GLP-1 analogues, DPP-4 inhibitors and SGLT2 inhibitors on the renal system.Diabetes and Vascular Disease Research. 2014 Sep; 11(5):306-23

17)Cooper ME. Pathogenesis, prevention, and treatment of diabetic nephropathy.The Lancet. 1998 Jul 18;352(9123):213-9

18) Taylor SI, Blau JE, Rother KI. SGLT2 inhibitors may predispose to ketoacidosis. The Journal of Clinical Endocrinology \& Metabolism. 2015 Aug 1; 100(8):2849-52.

19) Rosenstock J, Ferrannini E. Euglycemic diabetic ketoacidosis: a predictable, detectable, and preventable safety concern with SGLT2 inhibitors. Diabetes care. 2015 Sep 1; 38(9):1638-42.

20) Ogawa W, Sakaguchi K. Euglycemic diabetic ketoacidosis induced by SGLT2 inhibitors: possible mechanism and contributing factors. Journal of diabetes investigation. $2016 \mathrm{Mar}$; $7(2): 135$.

21) Halimi S, Verges B. Adverse effects and safety of SGLT-2 inhibitors. Diabetes \& metabolism. 2014 Dec 1; 40(6):S28-34.

22) Scheen AJ. Drug-drug interactions with sodium-glucose cotransporters type 2 (SGLT2) inhibitors, new oral glucoselowering agents for the management of type 2 diabetes mellitus. Clinical pharmacokinetics. 2014 Apr 1; 53(4):295-304. 